$5^{\mathrm{h}} 7^{\mathrm{m}} 3^{\mathrm{s}}+23^{\circ} 59^{\prime}$. Der Ort des helleren ist nach einer unter sehr ungünstigen Umständen Jan. 9 gemachten und daher wenig sicheren mikrometrischen Beobachtung, Anschluß an Berl B I692, angesetzt. Jan. 9 schätzte ich den helleren $10^{\text {m.o }}$ und $v={ }_{1} 1^{\mathrm{m}} \cdot 4$. Jan. 23 , Jan. 30 und Febr. 3 war $v$ an der

Düsseldorf, I 9 r 6 Febr. 23 .

$$
\mathrm{BD}+7^{\circ} 797 \quad 9^{\mathrm{m}} \cdot 44^{\mathrm{h}} 5^{8^{\mathrm{m}} 8^{\mathrm{s}} \cdot 4}+7^{\circ} 5^{8} \mathbf{3}^{\prime} .
$$

$\mathrm{SZ}_{325} \mathrm{Kr} \quad$ I854 Jan. 24, Luft sehr klar $9^{\mathrm{m}} \cdot 54^{\mathrm{h}} 58^{\mathrm{m}} 10^{\mathrm{s}} .0+7^{\circ} 59^{\prime} \cdot 3$

SZ $528 K r \quad 1854$ Sept. 27 , helle Dämmerung $9^{\mathrm{m}} \cdot 34^{\mathrm{h}} 5^{8^{\mathrm{m}}} 6^{\mathrm{s}} \cdot 7+7^{\circ} 57^{\prime} \cdot 6$

Es ist alles deutlich geschrieben und richtig berechnet. Der Stern erscheint verbürgt in $\mathrm{BD}$.

$\mathrm{BD}+18^{\circ} 23009^{\mathrm{m}} \cdot 39^{\mathrm{h}} 5^{\mathrm{I}^{\mathrm{m}} 26^{\mathrm{s}} \cdot 3}+{ }_{1} 8^{\circ} 54^{\prime} \cdot 9$.

SZ $624 K r_{1}{ }_{1855}$ Febr. I8, Luft gut $9^{\mathrm{m}} \cdot 59^{\mathrm{h}} 5 \mathrm{I}^{\mathrm{m}} 27^{\mathrm{s}} \cdot 5+\mathrm{I} 8^{\circ} 5^{\circ} 6^{\prime} 8$

SZ $639 K r \quad 1855$ April 6 , Luft meist sehr dunstig $9^{\mathrm{m}} \circ 9^{\mathrm{h}} 5^{\mathrm{I}^{\mathrm{m}}} 25^{\mathrm{s}} .0+18^{\circ} 53^{\prime} \circ$

Es ist auch hier alles deutlich geschrieben und richtig berechnet. Der Stern erscheint verbürgt in $B D$, wenn auch äußersten Sichtbarkeitsgrenze für den hiesigen Refraktor (Objektiv I $8.6 \mathrm{~cm}$ ) und demnach schwach I I ${ }^{\mathrm{m}} 5$. Mit Ermächtigung von Herrn Geheimrat Küstner teile ich im folgenden die mir von ihm gütigst übersandten Auszüge aus den Originalen der BD betreffend diese Sterne mit.

\section{W. Luther.}

die Differenz in Dekl. etwas groß ist. Bei beiden Sternen wird bis zur weiteren Aufklärung ein ? in BD hinzuzufügen sein. Vielleicht sind sie veränderlich.

$$
\mathrm{BD}+23^{\circ} 8829^{\mathrm{m}} \cdot 55^{\mathrm{h}} 7^{\mathrm{m}} 8 \mathrm{~s} \text {. }+23^{\circ} 5^{\prime \prime 2} \text {. }
$$

SZ ${ }_{7} 87$ Sch 1856 Jan. I, klare Luft $9^{\mathrm{m}} \cdot 55^{\mathrm{h}} 7^{\mathrm{m}} 6.7+23^{\mathrm{s}} 5^{8}$ : $^{\prime}$

Alles deutlich und richtig!

$$
\begin{aligned}
\text { SZ I } 75 K r \text { I } 857 \text { Febr. } 22 \text {, Luft sehr klar } \\
\qquad 9^{\mathrm{m}} \cdot 3 \quad 5^{\mathrm{b}} 7^{\mathrm{m}} 8^{\mathrm{s}} \cdot 7+23^{\circ} 57^{\prime} \cdot 9
\end{aligned}
$$

Hier ist aber der Grad verschrieben und muß $\mathbf{2}^{\circ}$ heißen, die Beobachtung gehört $\mathrm{zt}+22^{\circ} 867$. Der Stern $+23^{\circ} 882$ beruht dann nur auf der einzelnen Bestimmung in $\mathrm{SZ} 787$ und ist somit nicht gesichert. Wenn jetzt dort tatsächlich nichts am Himmel steht, so ist der Stern in BD zu streichen.

\title{
Bewegter Doppelstern in Lynx.
}

Bei der vergeblichen Suche nach dem Planeten I 9 I I LU wiederholte ich I916.I7 eine Gegend, die ich I903.05 aufgenommen hatte. Die Gegend, nahe dem parallaktischen Aquator gelegen, ist sehr reich an mäßig bewegten Sternen. Es fehlt mir die Zeit, sie jetzt zu behandeln; aber es kam mir ein so reizender bewegter Doppelstern von nahezu gleichhellen Komponenten in den Weg, daß ich mir nicht versagen kann, auf ihn aufmerksam zu machen.

Der Ort des Schwerpunktes des Systems, angeschlossen an $\mathrm{AG}$ Bonn 683 $\mathrm{I}$ und 686 $\mathrm{I}$ ist:

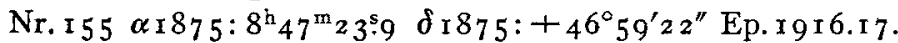
Das Paar ist gemeinsam um etwa $5^{\prime \prime}$ in den I 3 Jahren weiter gezogen. Da die Sterne der Umgebung fast alle wahrnehmbar

Königstuhl, I 9 r 6 März 3.

bewegt sind, so hält es schwer, die Bewegung einigermaßen absolut anzugeben. Für die südliche Komponente erhalte ich aus dem nächsten vorangehenden Stern ${ }^{1} 3^{\mathrm{m}} \cdot 5$ :

$$
\Delta s=0.396 \quad \varphi=245^{\circ}
$$

aus dem nächsten nördlichen Stern $\mathbf{I}^{\mathrm{m}}$ :

$$
\Delta s=0^{\prime \prime} 384 \quad \varphi=245^{\circ}
$$

während die nördliche Komponente in der Zwischenzeit sich eine Spur gegen die südliche verschoben $z u$ haben scheint, sodaß sich ihre Eigenbewegung $\Delta s=0^{\prime \prime}{ }_{3} 87$ bezw. O." 375 gegen die genannten Anschlußsterne ergibt.

Die beiden Komponenten sind etwa I I. Gr., die nördliche etwas schwächer. Die Komponenten stehen zur Zeit in 9.8 Distanz etwa unter dem P.-W. $135^{\circ}$.

\section{Wolf.}

\begin{tabular}{|c|c|c|c|c|c|}
\hline Nr. & Platte & Platt & enmitte & M. Z. Kgst. & Beob. \\
\hline I & $D \pm 355$ & $9^{h} 53^{m \cdot 8}$ & $+\mathrm{I} 2^{\circ} 5^{I^{\prime}}$ & $9^{\mathrm{h}} \times 5^{\mathrm{m}} \cdot \mathrm{o}$ & $M$. Wolf \\
\hline 2 & B $3^{699^{1}}$ ) & $12 \quad 17.9$ & +519 & II $17 \pm$ & $»$ \\
\hline 3 & $\mathrm{~B} 33^{\mathrm{I}} \mathrm{I}$ & o & + I $_{3}$ (etwa) & Io & F. Kaiser \\
\hline
\end{tabular}

\section{Mitteilungen über Kleine Planeten und Kometen.}

Aufnahmen auf der Königstuhl-Sternwarte.

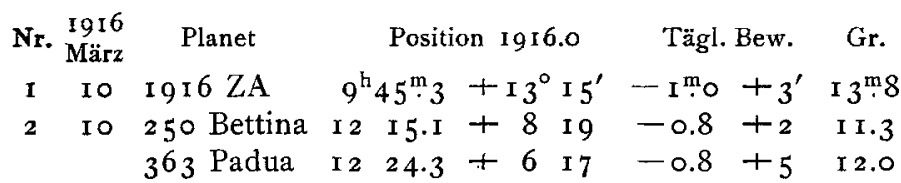

3 Auf einer älteren Platte von I 9 I 3 Febr. 5 fand sich noch 24 I Germania $756.6+16$ I I $-0.6-0 \quad$ I I.0

Heidelberg, I9I6 März I I.

M. Wolf.
Beobachtung des Kometen raise (Taylor). Auf der Sternwarte Utrecht von Observator $\mathcal{F}$. van der Bilt. I9I6 M.Z. Utrecht $\Delta \alpha \quad \Delta \delta \quad$ Vgl. Red.ad. l. app. Febr. 7 I $^{\mathrm{h}} 20^{\mathrm{m}} 6^{\mathrm{s}}+\mathrm{I}^{\mathrm{m}} 32^{\mathrm{s}} \cdot 3 \mathrm{x}+6^{\prime} 22^{\prime \prime} \mathrm{x}$ 1 $2,3+2^{\mathrm{s}} \cdot 22+7^{\prime \prime} \cdot 6$ app. $\alpha=5^{\mathrm{h}} 24^{\mathrm{m}} 36^{\mathrm{s}} .55\left(9.53^{2}\right)$, app. $\delta=+22^{\circ} 5^{\prime} 8_{1} 7^{\prime \prime} .5(0.729)$. Vergl.-Stern x 9 r6.0 $5^{\mathrm{h}} 23^{\mathrm{m}} 2^{\mathrm{s}} .02+22^{\circ}{ }_{5} \mathrm{I}^{\prime} 47^{\prime \prime} .8$ Berl B I $75^{2}$.

Beobachtungen des Kometen I916a (Neujmin).

Auf der Sternwarte Utrecht von Observator $\mathcal{F}$. van der Bilt. 1916 M. Z. Utrecht $\Delta \alpha \quad \Delta \delta \quad$ Vgl. Red.ad.1.app.

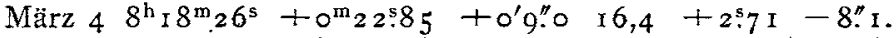
app. $\alpha=8^{\mathrm{h}} 59^{\mathrm{m}} 39^{\mathrm{s}} .85\left(9.23^{8}{ }_{\mathrm{n}}\right)$, app. $\delta=+\mathrm{I} \mathrm{I}^{\mathrm{o}} 37^{\prime} 25^{\prime \prime} \cdot 5(0.766)$. Vergl.-Stern I916.0 $8^{\mathrm{h}} 59^{\mathrm{m}} \mathrm{I} 4^{\mathrm{s}} .29+\mathrm{II}^{\circ} 37^{\prime} 24^{\prime \prime} 6$ Lpz I $3^{6} 3^{6}$. Am Lick Observatory, Mt. Hamilton, von Prof. $R$. Aitken. I 9 г 6 März $7.65^{8} 5$ Gr. app. $\alpha=9^{\mathrm{h}} \mathrm{I}^{\mathrm{m}} 3^{\mathrm{s}} \cdot 5$, app. $\delta=+9^{\circ} 53^{\prime} 20^{\prime \prime}$. (Telegramm an die Sternwarte Kopenhagen.)

Inhalt zu Nr. 4837. F. Fischer-Petersen. Über unendlich kleine retrograde, periodische Bahnen um die Massenpunkte im problème restreint. 20I. - 7. Voute. The parallax of the "Selected Area" region $19^{\mathrm{h}} \mathbf{2}^{\mathrm{m}}-59^{\circ} 41^{\prime}$. 203. - A. Abetti. Cometa $1913 \mathrm{f}($ Delavan). 205. - A. Abetti. Cometa 1915 a (Mellish). 207. - W. Luther. Beobachtungen des Veränderlichen Z Ceti. 209. - W. Luther. Neue Elemente und Maxima von Z Ceti. 2Ir. - H. Thiele. Über zwei Veränderliche im Orion. 213. - W. Luther. Bemerkungen zu drei Sternen der Bonner Durchmusterung. 2I3. - M. Wolf. Bewegter Doppelstern in Lynx. 215 Mitteilungen uber Kleine Planeten und Kometen. 2 I 5.

Geschlossen I9ı6 März 24. Herausgeber: H. Kobold. Druck von C. Schaidt. Expedition: Kiel, Moltkestr. 80. Postscheck-Konto Nr.6238 Hamburg II. 